Rechtsmedizin $2011 \cdot 21: 257-257$

DOI 10.1007/s00194-011-0774-9

Online publiziert: 14. Juli 2011

(c) Springer-Verlag 2011

\author{
H. Bratzke \\ Institut für Rechtsmedizin, Klinikum der Johann Wolfgang \\ Goethe-Universität, Frankfurt am Main
}

\title{
8. International Symposium Advances in Legal Medicine/90. Jahrestagung der Deutschen Gesellschaft für Rechtsmedizin
}

\author{
26.09. bis 01.10.2011 in Frankfurt a. M.
}

Liebe Leserinnen und Leser,

mit diesem Heft begrüßen wir besonders herzlich die Teilnehmer der 8. International Symposium Advances in Legal Medicine (ISALM) Conference in Frankfurt a. M., die zum vierten Mal in Deutschland stattfinden wird (Berlin 1993, Mainz 1999, Hamburg 2005). Aber nicht nur unsere Freunde aus Japan sind in großer Zahl in den neuen Universitätscampus im Westend Frankfurts gekommen, sondern zahlreiche weitere Gäste aus Europa, Asien und „vom anderen Ende der Welt" aus Neuseeland und Australien.

Mit über 120 Vorträgen und 150 Postern wurde ein anspruchsvolles wissenschaftliches Programm zusammengestellt, das die aktuellen Entwicklungen in der Rechtsmedizin repräsentiert.

Besonders erfreulich ist wieder der deutsch-japanische Juristentag, der sich mit den Themen „Probleme der Transplantation“ und „Kindesmisshandlung“ in den verschiedenen Ländern aus juristischer Sicht beschäftigen und sehr ertragreich sein wird.

Nicht zu vergessen ist die 90. Jahrestagung der Deutschen Gesellschaft für Rechtsmedizin, die mit einem „Deutschen Tag“ in die ISALM eingebettet ist. Eine der Sitzungen ist dem Gedenken an den kürzlich verstorbenen Altmeister der Rechtsmedizin, Herrn Prof. Steffen Berg, gewidmet.

Der Kongress findet auf dem WestendCampus statt, dessen Gebäude eine wechselvolle Geschichte hinter sich haben. Das Hauptgebäude wurde nach Entwürfen von Hans Poelzig als Zentralverwaltung für die
I.G. Farben von 1928 bis 1931 errichtet und diente nach Kriegsende als Hauptquartier der amerikanischen Armee. Seit 2001 sind zahlreiche Fachbereiche der Johann Wolfgang Goethe-Universität Frankfurt a. M. in den „Poelzig-Bau“ umgezogen, und auf dem Campus sind attraktive Gebäude für die Wirtschafts- und Rechtswissenschaften errichtet worden.

Die Verbindung von Traditionellem mit Modernem zeigt sich auch in den vielfältigen wissenschaftlichen Beiträgen, deren Abstracts im vorliegenden Heft der Rechtsmedizin abgedruckt sind. Die aktuellen Entwicklungen in der molekularen Medizin haben vor der Rechtsmedizin nicht Halt gemacht, und Ursachen des plötzlichen Herztodes werden nunmehr auch auf molekularer Ebene bestimmt. In der Toxikologie stehen genetische Einflüsse auf Verstoffwechselung, Toxizität, Gewöhnung und Missbrauch im Fokus der Wissenschaft, und ohne Weiterentwicklung und Forschung auf diesem Gebiet wird die forensische Toxikologie nicht auskommen.

Dass Deutschland auf diesen Gebieten mit seinen universitären Instituten weltweit hohe Anerkennung genießt und anderen Ländern zum Vorbild dient, darf nicht darüber hinwegtäuschen, dass im eigenen Land der Rechtsmedizin das Leben mehr als schwer gemacht wird. Als interdisziplinäres Fach sitzt es im wahrsten Sinne des Wortes zwischen allen Stühlen, v. a. wenn es um die Verantwortung für die Finanzierung des wissenschaftlichen Personals und Investitionen in Geräte und Gebäude geht. Postmortale bildgebende Verfahren (Computertomographie, Magnetresonanztomo- graphie) sind z. B. in der Schweiz fast schon zum Standard geworden, valide toxikologische Untersuchungen ohne „liquid chromatography-mass spectrometry-mass spectrometry“ (LC-MS-MS) nicht denkbar, und die Sequenzierung genetischer Marker gehört zum modernen Rüstzeug forensischer Molekularbiologie.

Wir hoffen, dass mit diesem internationalen Kongress bei den Verantwortlichen das Bewusstsein geweckt wird, dass man die führende Rolle der deutschen Rechtsmedizin nicht unnötig verspielen sollte. Erst kürzlich ist der deutschen Rechtsmedizin durch die Zuerkennung eines Impact-Faktors in Höhe von 0,876 für die vorliegende Zeitschrift Rechtsmedizin die hohe Wertschätzung dokumentiert worden, und das International Journal of Legal Medicine gehört zu den weltweit meistzitierten Zeitschriften in unserem Fach.

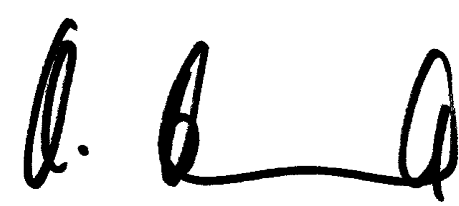

Prof. Dr. Hansjürgen Bratzke

\section{Korrespondenzadresse Prof. Dr. H. Bratzke}

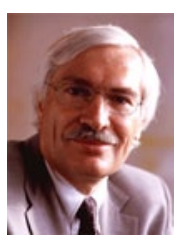

Institut für Rechtsmedizin, Klinikum der Johann Wolfgang Goethe-Universität Kennedyallee 104, 60596 Frankfurt am Main bratzke@em.uni-frankfurt.de 